\title{
Facile Synthesis, Metastable Phase Induced Morphological Evolution and Crystal Ripening, and Structure-Dependent Photocatalytic Properties of 3D Hierarchical Anatase Superstructures
}

\author{
Guoliang Li, ${ }^{* \dagger}$ Qiuwen Chen, ${ }^{* \dagger, \S}$ and Jing $\operatorname{Lan}^{*}$ \\ ${ }^{\dagger}$ State Key Laboratory of Environmental Aquatic Chemistry, Research Center for Eco-Environmental Sciences, Chinese Academy of \\ Sciences, Beijing 100085, People's Republic of China \\ ${ }^{\ddagger}$ College of Chemistry and Pharmaceutical Sciences, Qingdao Agricultural University, Qingdao 266109, People’s Republic of China \\ ${ }^{\S}$ Center for Eco-environmental Research, Nanjing Hydraulic Research Institute, Nanjing 210029, People’s Republic of China
}

Supporting Information

\begin{abstract}
Unique 3D hierarchical anatase $\mathrm{TiO}_{2}$ superstructures with evolvable morphologies and tunable sizes were successfully fabricated through a facile solvothermal route without using any structure-directing additives. A complex assembly process involving nucleation of nascent nanoparticles, agglomeration and ordered attachment of tiny nanocrystals, and regrowth induced by metastable crystal phase was put forward for the formation and morphology evolution of the anatase superstructures. It was revealed the crystalline phase experienced a ripening process from semi-

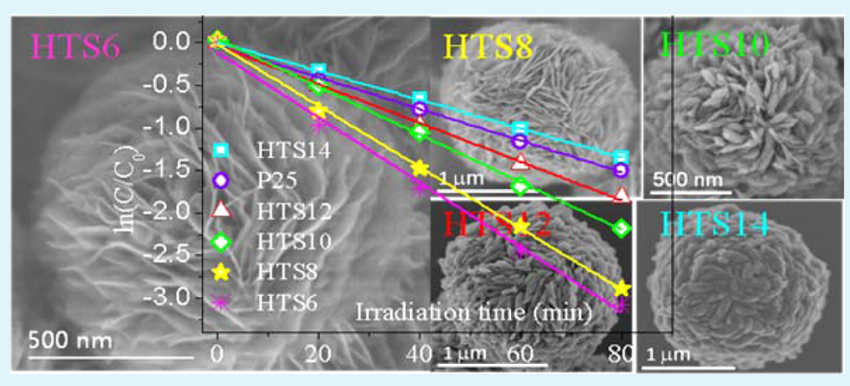
crystal to mesocrystal status accompanying the morphologies evolving from peony-like to chrysanthemum-like and eventually to spherical structures. The obtained 3D hierarchical anatase $\mathrm{TiO}_{2}$ superstructures exhibited superior photocatalytic activities for organic pollutant degradation, which could be largely attributed to the more efficient light-harvesting ability and the high specific surface area of the unique structures.
\end{abstract}

KEYWORDS: 3D hierarchical superstructures, titania, evolution, ripening, photocatalysis

\section{INTRODUCTION}

Research aimed at constructing inorganic hierarchical superstructures assembled by nanoscale subunits has recently become an attractive trend in nanosciences and nanotechnology, not only due to their amazing versatility sprung from different monomorphological nanostructures but also to the widely recognized fact that properties and functions of nanomaterials depend considerably on the morphology, structure, phase, shape, size, and dimensionality. ${ }^{1-5}$ Particularly, 3D hierarchical structures, which are organized from $1 D$ or $2 \mathrm{D}$ nanobuilding units, have attracted rapidly increasing attention, due to their potential and promising applications in photocatalysis, separation, adsorption, sensors, electronics, dyesensitized solar cell, and so on. ${ }^{6-11}$ Many methods have been developed for preparing these structures, of which one-pot template-free hydrothermal/solvothermal procedures are more preferable and recommendable than commonly adopted template ones, due to their simplicity and adjustability. Up to now, a variety of $3 \mathrm{D}$ superstructures have been acquired in a delicately controlled manner. However, developing facile approaches for controllable fabrication of evolvable 3D superstructures remains a great challenge. What is more, to our knowledge, almost all the publications just focus on fabrication of a defined structure, there is still no work penetrating into what would happen to the products if the hydrothermal/solvothermal process continued.

As one of the most promising semiconductor materials with numerous practical applications, titanium dioxide $\left(\mathrm{TiO}_{2}\right)$ has been extensively investigated. ${ }^{12-17}$ To date, $\mathrm{TiO}_{2}$ with various morphologies has been synthesized, such as particles, rods, tubes, spheres, etc., of which $3 \mathrm{D}$ hierarchical nanostructures are considered one of the most attractive photocatalyst materials. ${ }^{18-23}$ On the one hand, hierarchical superstructures usually possess larger surface area, well-facilitating adsorption and photoreaction of reactants. ${ }^{24,25}$ On the other hand, they have the advantages of more efficient light harvesting ability due to multireflection of irradiated light within the voids and between the subunits of the materials, therefore obtaining higher photoefficiency. $^{24,26}$ In addition, the existence of a great number of sharp edges and corners in the 3D hierarchical superstructures is in favor of the movement of electrons and holes in semiconductors, improving the photochemical and catalytic performance of $\mathrm{TiO}_{2} \cdot{ }^{27}$ Although very recently several groups reported synthesis of $3 \mathrm{D}$ hierarchical $\mathrm{TiO}_{2}$ with improved photocatalytic activities, ${ }^{8,28,29}$ they usually limited

Received: September 29, 2014

Accepted: December 1, 2014

Published: December 1, 2014 
either in adopting routes of complicated procedures, or in obtaining products of defined morphology and lower surface area. ${ }^{28,30}$ Therefore, it is still an urgent need to develop versatile synthetic process for $3 \mathrm{D}$ hierarchical $\mathrm{TiO}_{2}$ superstructures with evolvable morphology. Meanwhile, it is also a pity no light, to our knowledge, has been cast on postformation evolution of $3 \mathrm{D}$ hierarchical $\mathrm{TiO}_{2}$ superstructures.

To tackle these problems, we propose here a strategic solvothermal procedure for synthesis of $3 \mathrm{D}$ hierarchical $\mathrm{TiO}_{2}$ superstructures (HTSs) self-organized from ultrathin $\mathrm{TiO}_{2}$ nanosheets and penetrate into morphology evolution of samples in the postformation process (Scheme 1). A complex

\section{Scheme 1. Schematic Illustration of the Morphological} Evolution

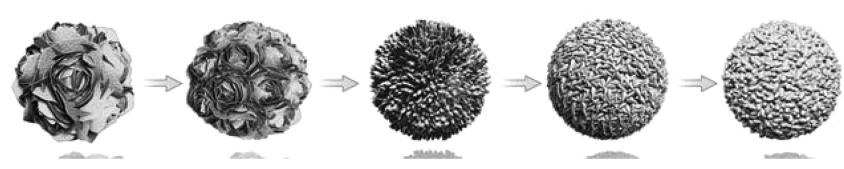

assembly process involving nucleation of nascent nanoparticles, agglomeration and ordered attachment of tiny nanocrystals, and regrowth induced by metastable crystal phase was put forward for the formation and morphology evolution of the anatase superstructures. Moreover, superior photocatalytic performance for degradation of organic pollutant was also demonstrated for the obtained 3DHTSs.

\section{EXPERIMENTAL SECTION}

Preparation of HTSs. The detailed composition of the solutions and the solvothermal reaction conditions for synthesizing HTSs with different structures are listed in Table $\mathrm{S} 1$ in the Supporting Information. In a typical experimental procedure, $0.74 \mathrm{~g}$ of $\mathrm{NH}_{4} \mathrm{~F}$ was added into $5 \mathrm{~mL}$ deionized water and magnetically stirred until completely dissolved, followed by addition of $50 \mathrm{~mL}$ ethylene glycol (EG). After the mixture was stirred for $15 \mathrm{~min}, 5 \mathrm{mmol} \mathrm{TiOSO}_{4}$ was added into the solution during strong stirring. Then, the mixture solution was transferred into a $100 \mathrm{~mL}$ Teflon pot and sealed tightly in a stainless steel bomb and heated at $180{ }^{\circ} \mathrm{C}$ for $6 \mathrm{~h}$ at an increase rate of $3 \mathrm{~K} \mathrm{~min}^{-1}$. The products of the solvothermal reaction were washed with absolute ethanol and distilled water three times, respectively. The
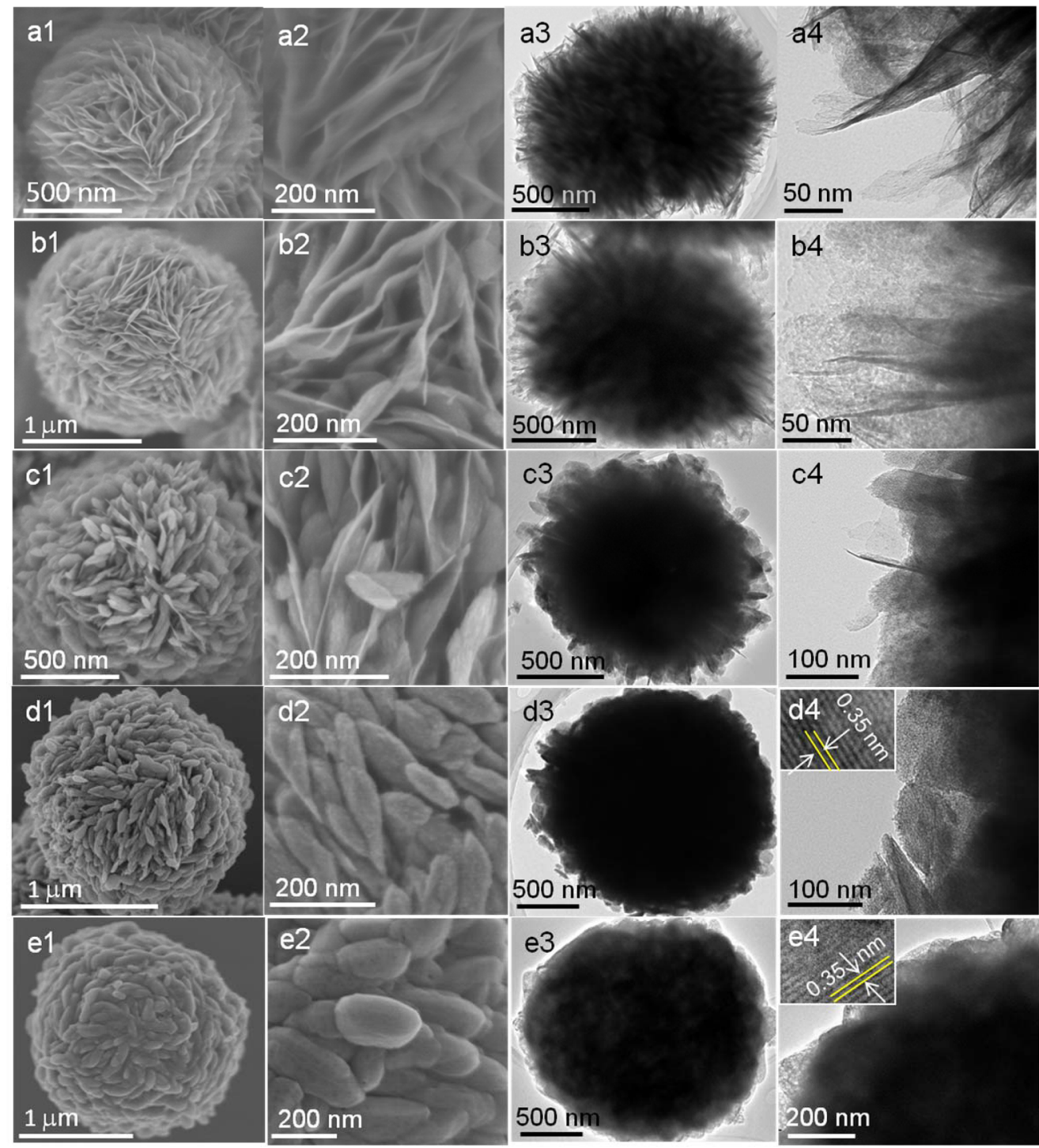

Figure 1. SEM and TEM images of the HTSs obtained with varying reaction time: HTS6 (a1-a4); HTS8 (b1-b4); HTS10 (c1-c4); HTS12 (d1$\mathrm{d} 4$, the inset in panel d4 shows a high-resolution TEM image of the paddy particle); HTS14 (e1-e4, the inset in e4 shows a high-resolution TEM image of the rice particle). Note: SEM images were taken without sputter coating. 
white precipitates were collected and vacuum dried at $80{ }^{\circ} \mathrm{C}$ overnight and kept in a desiccator. A portion of powders were calcined at $500{ }^{\circ} \mathrm{C}$ for $1 \mathrm{~h}$ for further characterization and application use.

Characterization. The morphology of the HTSs was obtained with a field-emission electron microscope (SU8020, Hitachi, Japan) with an accelerating voltage of $3 \mathrm{kV}$. The samples were free of pretreatment by metal sputter coating. Higher resolution transmission electron microscopy (HRTEM) and the selected area electron diffraction (SAED) patterns of the samples were obtained on a transmission electron microscope (JEOL, JEM-2100, Japan) operated at $200 \mathrm{kV}$. The X-ray diffraction (XRD) patterns were recorded with a PANalytical X'Pert Pro X-ray diffractometer (PANalytical, The Netherlands) equipped with $\mathrm{Al} \mathrm{K} \alpha$ radiation $(40 \mathrm{kV}, 200 \mathrm{~mA}$ ). Nitrogen adsorption-desorption isotherms were measured with a Micromeritics ASAP2000 V3.01 analyzer. The Brunauer-EmmettTeller (BET) specific area was calculated using the BET equation. The pore size distribution was obtained using the Barret-Joyner-Halenda (BJH) equation. The thermogravimetric/differential thermal analysis (TG/DSC) was examined on a Netzsch STA449 instrument from room temperature to $800{ }^{\circ} \mathrm{C}$ with a heating rate of $10 \mathrm{~K} \mathrm{~min}^{-1}$. The $\mathrm{X}$ ray photochemical spectra was performed on a Thermo SCIENTIFIC ESCALAB 250 equipped with Al-Ka alpha radiation. UV-vis diffuse reflectance spectra (DSR) was performed on a Hitachi U4100 spectrometer.

Photocatalytic Activity. The photocatalytic activities of the 3D HTSs were examined by degradation of azo-dye Rhodamine B (Rh.B) at room temperature in a custom-made $100 \mathrm{~mL}$ reactor. An $8 \mathrm{~W}$ mercury lamp with a characteristic wavelength of $365 \mathrm{~nm}$ was placed beside the reactor as a light source. $0.01 \mathrm{~g}$ HTSs were added into 50 $\mathrm{mL}$ Rh.B solutions $\left(2 \mathrm{mg} \mathrm{L}^{-1}\right)$ in the reactor and magnetically stirred in the dark at a speed of $800 \mathrm{rpm}$ for $1 \mathrm{~h}$ to ensure the adsorption equilibrium and eliminate the diffusion effects. Then, the mixture solution was irradiated. Samples were taken at an interval of $20 \mathrm{~min}$, filtered through a $0.2 \mu \mathrm{m}$ cellulose acetate membrane and detected by UV-vis spectroscope (Hitachi U4100, Japan). A pseudo-first-order reaction rate equation was used to evaluate the degradation kinetics for HTSs. The equation was defined as $\ln \left(C / C_{0}\right)=k t$, where $C$ and $C_{0}$ refer to the concentration of Rh.B at the irradiation time $t$ and $0 \mathrm{~min}, k$ and $t$ represent the reaction rate constant and photodegradation time, respectively.

\section{RESULTS AND DISCUSSION}

To monitor the morphology evolution and crystallization process from unstable to fully crystallized condition in the postformation period, an investigation using SEM, TEM, XRD, and thermogravimetric/differential thermal analysis (TG/DSC) was conducted. During the hydrothermal condition, nanoparticles were first formed as nucleation seeds (2h, Figure S1a1, Supporting Information). Simultaneously, some nanoparticles aggregated forming fluffy structures (Figure S1a2, Supporting Information). With the increase of the hydrothermal reaction time to $3 \mathrm{~h}$, the nanoparticles that constructed the fluffy structures experienced an orderly attachment of crystallite forming rudimentary loosely compact nanosheets (Figure S1b, Supporting Information). Further increasing the reaction time to $6 \mathrm{~h}$, we obtained integral flower-like superstructures (Figure S1c, Supporting Information). Figure 1 shows electron micrographs depicting the subsequent integral structure evolution with reaction time (prior to calcination). As shown in Figure 1a, peony-like structures of big petals with an average diameter of $800 \mathrm{~nm}$ were obtained after a $6 \mathrm{~h}$ solvothermal reaction, which were composed of randomly oriented ultrathin nanosheets with a thickness of several nanometers. The petals appear mesh-like textures with pores strewed (Figure S2, Supporting Information). An obvious diffraction peak around $2 \theta=25.24^{\circ}$ appeared in the corresponding XRD pattern (Figure 2a), demonstrating semicrystal nature that endows

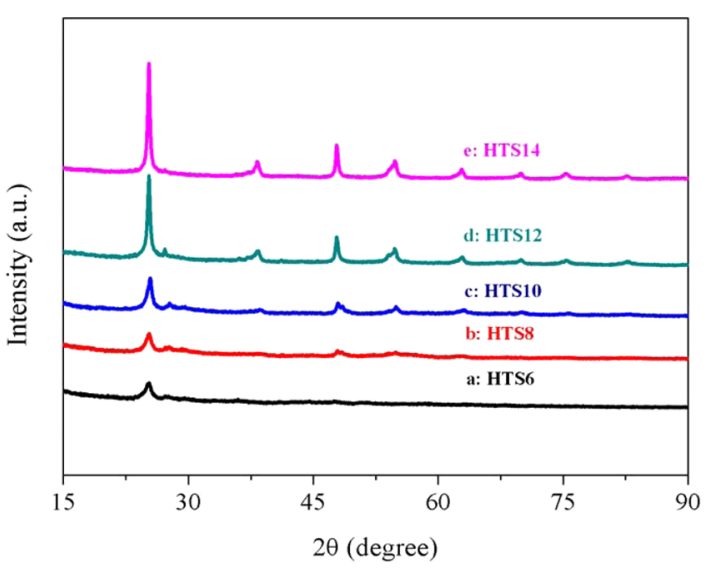

Figure 2. XRD patterns of the HTSs obtained with varying reaction time: HTS6 (a), HTS8 (b), HTS10 (c), HTS12 (d), and HTS14 (e).

HTS6 with the possibility to further grow and evolve with continuing solvothermal reaction. After $2 \mathrm{~h}$, HTS 8 retained the hierarchical peony-like structure (Figure $1 \mathrm{~b}$ ), which was also composed of ultrathin mesh-like nanosheets, but petals of the peony became smaller and denser (Figure 1bl). The XRD pattern (Figure $2 \mathrm{~b}$ ) had three main diffraction peaks around $2 \theta$ $=25.24,48.23$, and $55.29^{\circ}$, all of which can be ascribed to the formation of anatase titanium oxide (JCPDS No.70-6826). After two more hours of solvothermal treatment (HTS10), the peony-like morphology evolved to chrysanthemum-like morphology with the large sheet petals evolving to thin willow-leaf like petals, and the overall structure became compact (Figure 1c) and larger (Figure S3, Supporting Information). The corresponding XRD pattern demonstrates the more crystalline nature of the titanium oxide (Figure 2c), in which an increased peak intensity in the anatase titania phase and the occurrence of additional diffraction peaks around $2 \theta=25.24,38.0,48.3,54.5$, and $62.8^{\circ}$ were observed. These peaks can be indexed to the (101), (103), (200), (105), and (213) planes of the anatase titania, respectively, indicating a ripening crystallizing process in the $2 \mathrm{~h}$. With the reaction time increased to $12 \mathrm{~h}$, petals of the chrysanthemum-like spheres tended to curl and compact, forming paddy-like particles (Figure 1d). The paddy-like particles have a lattice fringes spacing of about $0.35 \mathrm{~nm}$ (inset in Figure 1d4), corresponding to the (101) plane of the anatase titania. An increased peak intensity in the XRD pattern of HTS12 (Figure 2d) around $2 \theta=25.24,38.0,48.3,54.5$, and $62.8^{\circ}$ was observed, confirming the deepening crystallinity of the product. When the reaction time was further increased to $14 \mathrm{~h}$, the morphology of the product became condensed spheres composed of nanoscale rice-like particulate $\mathrm{TiO}_{2}$ crystals (Figure 1e). The corresponding XRD pattern (Figure 2e) reveals these spheres were well-crystallized anatase phase titania. What is more, SAED patterns of these HTSs appear different. Both HTS6 and HTS8 exhibit a semicrystalline phase (Figure S4a,b, Supporting Information). HTS10 shows rings in the SAED pattern (Figure S4c, Supporting Information), indicating a random orientation between the crystallites. The SAED pattern of HTS12 (Figure S4d, Supporting Information) shows more dots with minor broadening, indicative of a stronger alignment between the crystallites. HTS14 exhibits a monocrystal SAED pattern (Figure S4e, Supporting Information), which demonstrates crystallites or particles in HTS14 are highly ordered aligned. 
Such prepared HTSs possess mesoporous networks proved by the $\mathrm{N}_{2}$ adsorption-desorption analysis (Figure 3 ). Specific
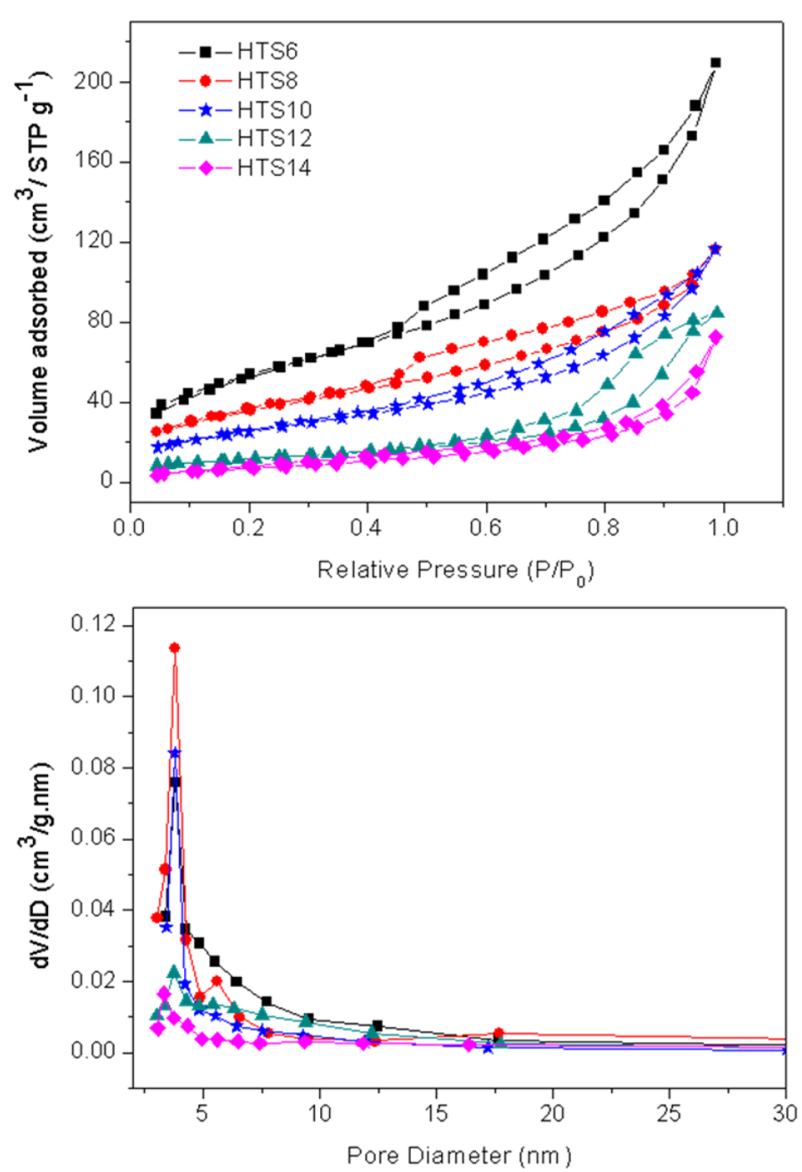

Figure 3. $\mathrm{N}_{2}$ adsorption-desorption isotherms and $\mathrm{BJH}$ pore size distribution curves for HTSs.

surface area and pore volume exhibited a tendency to decrease from 197 to $23 \mathrm{~m}^{2} \mathrm{~g}^{-1}$ and 0.32 to $0.027 \mathrm{~cm}^{3} \mathrm{~g}^{-1}$, respectively, when reaction time increased from 6 to $14 \mathrm{~h}$ (Table S2, Supporting Information). This result corresponds to the morphology evolution from ultrathin nanosheets based hierarchical structures to larger and condensed spheres. Such porous structures can provide more efficient transport pathways for molecules, which is critical for catalyst and other applications. More importantly, such unique 3D hierarchical structures allow multireflections of irradiated light sources, such as ultraviolet light, within their cross-linked structures, maximizing light harvesting and thus increasing quantities of photogenerated electrons and holes functioning in the photocatalytic reaction, which endows HTSs with greatly enhanced photocatalytic activity.

The increased maturity of the crystalline nature of the products corresponding to the increased solvothermal reaction time can be further supported by TG/DSC (Figure S5, Supporting Information). It can be observed from the DSC results that a sharp exothermic peak centered at $490{ }^{\circ} \mathrm{C}$ appeared for HTS8, which resulted from the crystallization from amorphous to anatase phase. With prolonging of the solvothermal reaction, this exothermic peak became less defined due to decrease of the amorphous species in the products. Especially for HTS14, there is no obvious exothermic peak observed on the corresponding DSC curve, which indicates the amorphous species in the HTS14 has been fully converted to anatase phase. TGA curves also reveals the overall weight loss of the samples decreased with the increase of the solvothermal treatment time, indicating the samples became less hydrated and the organic species content decreased with increasing solvothermal treatment time.

On the basis of the above-mentioned experimental results, e.g., SEM, TEM, XRD, and TG/DSC, the morphological evolution mechanism throughout the crystal growth from formation of HTS6 to its postformation process can be proposed as described in following steps: (1) nucleation of small nanoparticles and the simultaneous aggregation. At this stage, large numbers of nanoparticles produced upon solvothermal reaction initially induces a high collision frequency leading to a random agglomeration of particles, which have open porous structure and therefore contribute little to reduction of surface area per unit volume; (2) ordered attachment of particles or crystallites form hierarchical superstructures. With the decrease of particle number density and collision frequency, the particles start to aggregate in a more ordered fashion, eliminating the solution-solid interface to minimize the system free energy; (3) renucleation and regrowth in the postformation period. As a model for nanoparticle aggregation proposed by Nomura et al., ${ }^{31}$ when a significant number of "ordered" attachments has taken place, the "released" ions may be numerous enough to induce a second nucleation process. During this stage, larger ultrathin nanosheets split to smaller ones and further evolved to ricegrain like particles to eliminate the smaller edge faces, which was induced by both the slow desaturation or ripening and their hydrophobic nature of high energy.

To understand the role of reagents composition in the formation of 3DHTS, a series of control experiments were conducted (Table S1, Supporting Information). As shown in Figure S6 (Supporting Information), the resulting products appeared as varied morphologies in the case of different reagents compositions. Anomalous spherical particles were formed with an increased dose of $\mathrm{H}_{2} \mathrm{O}$ to $10 \mathrm{~mL}$ (Figure S6a, Supporting Information). It was unformed smaller nanoparticles that were obtained if the dose of $\mathrm{H}_{2} \mathrm{O}$ was further increased to $20 \mathrm{~mL}$ (Figure S6b, Supporting Information). What is more, the morphologies of the products also vary with changes of the $\mathrm{NH}_{4} \mathrm{~F}$ amount. The morphologies appeared to be nanoparticles (Figure S6c, Supporting Information), evolving spheres (Figure S6d, Supporting Information), or octangular plates (Figure S6e, Supporting Information) when $\mathrm{NH}_{4} \mathrm{~F}$ was absent, decreased to $0.5 \mathrm{~g}$, or increased to $1 \mathrm{~g}$, respectively. What is more, if $\mathrm{NH}_{4} \mathrm{~F}$ was substituted by other reagents like $\mathrm{NH}_{4} \mathrm{Ac}$ and $\mathrm{NH}_{4} \mathrm{Cl}$, no hierarchical structures but rather amorphous products were formed. Substitution of $\mathrm{NH}_{4} \mathrm{~F}$ with $\mathrm{NaF}$ can also result in the formation of the hierarchical structures, but the samples were not as integral and wellproportioned as those for $\mathrm{NH}_{4} \mathrm{~F}$. Therefore, $\mathrm{NH}_{4} \mathrm{~F}$ plays an important role in formation of the unique hierarchical structures. In addition, replacing the $\mathrm{TiOSO}_{4}$ with other $\mathrm{Ti}$ precursors, e.g., titanium butoxide and titanium isopropoxide, no hierarchical superstructures but rather only hexahedral sticks and plates were obtained (Figure S6f,g, Supporting Information). Therefore, it is reasonable to propose that acidic conditions also function in forming 3DHTS. Under weak acidic conditions of the solvothermal reaction, fluorine ions preferentially bind to the higher energy facets, e.g., $\{001\}$ facets, of the initially formed nanoparticles and restraining the growth 

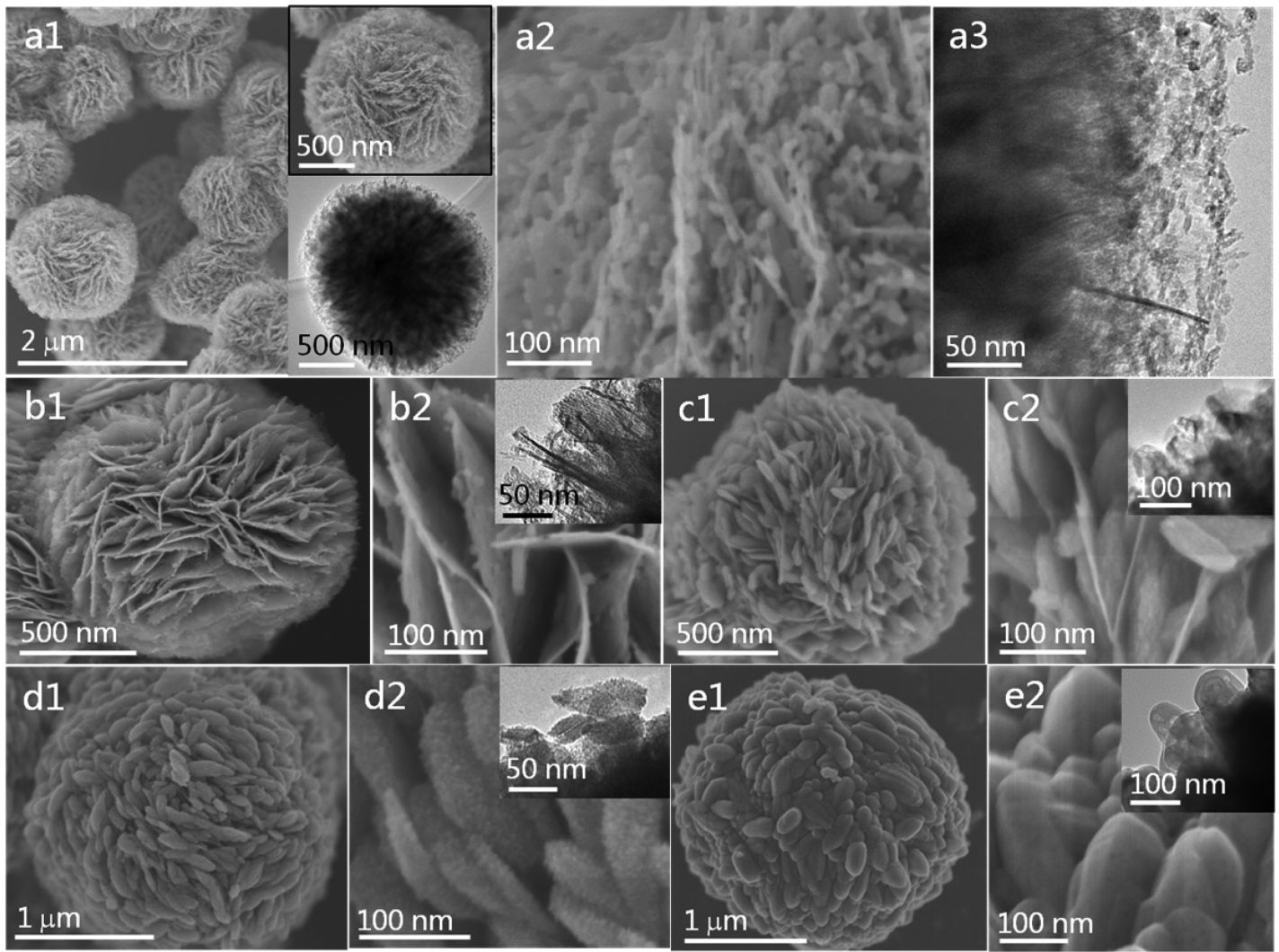

Figure 4. SEM and TEM images of the HTSs samples calcined at $500{ }^{\circ} \mathrm{C}$ for 1 h: HTS6 (a1-a3); HTS8 (b1-b2); HTS10 (c1,c2); HTS12 (d1,d2); HTS14 (e1,e2).

along the corresponding direction, ${ }^{9}$ thus leading to formation of nanosheet petals and the subsequent construction of hierarchical structures under combined effects with other factors. It is also worth mentioning that in replacing EG with absolute ethanol, no hierarchical peony-like superstructures were obtained and only resulted in rectangular grains (Figure S6h, Supporting Information). Increasing the $\mathrm{H}_{2} \mathrm{O}$ ratio results in faster hydrolyzation of $\mathrm{TiOSO}_{4}$, therefore leading to smaller nanoparticles. Combined with the fact that an increased dose of $\mathrm{H}_{2} \mathrm{O}$ also confined the product in simple shape like nanoparticles, it is reasonable to propose the viscosity of the reaction system plays an important role in formation of the superstructure. ${ }^{32}$ Either replacing EG with ethanol or increasing the ratio of $\mathrm{H}_{2} \mathrm{O}$ has a common effect of markedly decreasing the viscosity of the reaction system, which acted against the aggregation of nanosubunits for constructing hierarchical superstructures.

The semicrystalline peony-like and chrysanthemum-like samples of HTS6, HTS8, and HTS10 can be converted to the fully crystalline anatase phase by calcination at $500{ }^{\circ} \mathrm{C}$ for 1 $\mathrm{h}$ in air without changing the overall morphologies. As illustrated in Figure 4, HTS6 remains its peony-like structure (Figure 4a1), but the subunits of nanosheets become coarser with nanoparticles strewing all over the nanosheets (Figure $4 a 2,3)$. This can be ascribed to the removal of a large amount of organic species (Figure S5, Supporting Information, TGS) by calcination. Different from HTS6, subunits nanosheets of HTS8 are smoother with little change (Figure 4b2,3). The chrysanthemum-like HTS10 also went through the similar experience as that of HTS8. The calcined counterpart also remains the similar overall morphology, but the subunits petals of chrysanthemum did not convert to mesh-like structure as HTS6, just became coarser due to its lower content of organic species. As for HTS12 and HTS14, Figure 4d,e clearly shows that they remain unchanged in morphology after calcination due to their high crystalline nature.

The crystal phase and crystallinity of the calcined HTSs were revealed by XRD analysis. As shown in Figure S7 (Supporting Information), all the XRD patterns can be assigned to the tetragonal anatase phase of titania (JCPDS No. 21-1272), exhibiting high purity nature after calcination. The diffraction peaks became sharper when the solvothermal reaction time was prolonged from 6 to $14 \mathrm{~h}$, indicating formation of larger anatase crystals with enhanced crystallinity by increasing the solvothermal reaction time. In addition, surface composition and chemical status of the calcined HTSs were investigated by X-ray photoelectron spectroscopy (XPS, Figure S8, Supporting Information). The binding energy of $\mathrm{Ti} 2 \mathrm{P}_{3 / 2}$ and $\mathrm{Ti} 2 \mathrm{P}_{1 / 2}$ is around 458.5 and $464.3 \mathrm{eV}$, respectively, indicating the oxidation state of the Ti element is the same as that of bulk $\mathrm{TiO}_{2}$. The high-resolution XPS spectrum of $\mathrm{F} 1 \mathrm{~s}$ indicates that the measured binding energy is $684.1 \mathrm{eV}$, which is the typical binding energy of fluorated $\mathrm{TiO}_{2}$ systems, such as $\equiv \mathrm{Ti}-\mathrm{F}$ species on $\mathrm{TiO}_{2}$ crystal surfaces. Because the binding energy of $\mathrm{F}$ 1s for atomic incorporation of $\mathrm{F}$ atoms or their substitution for $\mathrm{O}$ atoms is $688.5 \mathrm{eV},{ }^{33,34}$ such results indicate the F element in the product is present as surface atoms. The carbon peak is attributed to the residual carbon of the product and the adventitious hydrocarbon from the XPS instrument itself.

To demonstrate structure-function correlation of the prepared HTSs, photocatalytic activities of the calcined samples were examined using decolorization of azo-dye Rhodamine B 
(Rh.B) as a probe reaction. Degussa P25, a well-known highly efficient commercial $\mathrm{TiO}_{2}$ photocatalyst, was used for comparison. As shown in Figure S9 (Supporting Information), HTS6 exhibits the highest photocatalytic activity of nearly $100 \%$, whereas other samples show significantly lower activity. In particular, the compact rice-grain constructed sphere (HTS14) presents an activity as low as $72 \%$. Activity of P25 is similar to that of HTS14 (74\%). To better understand the photocatalytic activity of the HTSs, the reaction kinetics of the Rh.B degradation was performed using a pseudo-first-order reaction rate equation $\ln \left(C / C_{0}\right)=k t$. As shown in Figure 5 ,

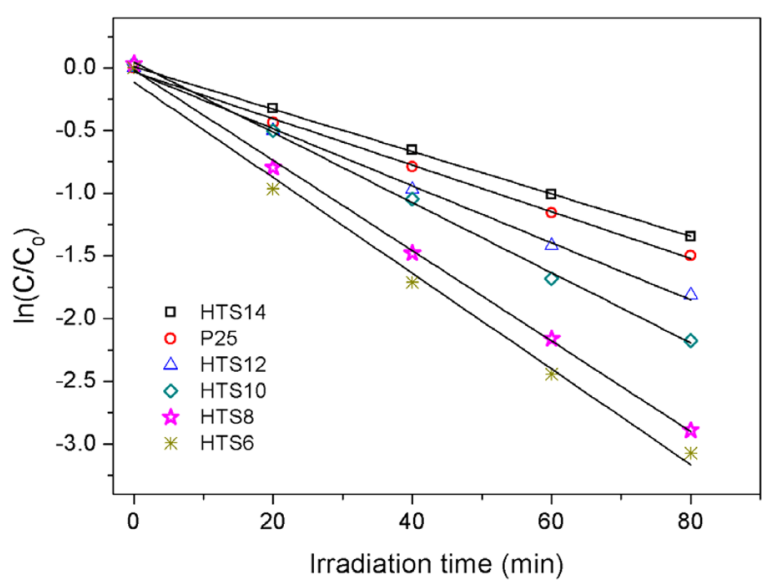

Figure 5. Photocatalytic degradation of Rhodamine B using calcined HTSs samples and Degussa P25 nanoparticles.

plots for all the samples appear linear indicating they fit well with the pseudo-first-order reaction kinetics. The degradation rate of Rh.B decreased with increasing solvothermal reaction time (from 6 to $14 \mathrm{~h}$ ). The hierarchical peony-like HTS6 exhibit a much higher degradation rate $\left(k=0.038 \mathrm{~min}^{-1}\right)$ than those samples of longer solvothermal times, which are 0.036 , $0.028,0.023$, and $0.017 \mathrm{~min}^{-1}$ for HTS8, HTS10, HTS12 and HTS14, respectively. The degradation rate of Degussa P25 is $0.019 \mathrm{~min}^{-1}$, which is just relevantly faster than that of HTS14.

It is known that there have been four strategies reported to improve the photocatalytic activity of $\mathrm{TiO}_{2}:{ }^{35}$ (1) improving light-harvesting efficiency by multiple scattering/reflection; ${ }^{23,24,29}$ (2) increasing the specific surface area through preparing various photocatalysts of microporous and macro-/ mesoporous $\mathrm{TiO}_{2}{ }^{36,37}$ (3) forming a rutile/anatase junction to improve the separation of the photogenerated $\mathrm{e}-\mathrm{h}$ pairs; ${ }^{38,39}$ (4) controlling the exposed facets, e.g., $\{001\}$, of the $\mathrm{TiO}_{2}$ crystal. But there are still controversies reported in the latter two strategies. $5,11,35$ Because all these HTSs and the P25 possess a similar crystalline nature. The superior photocatalytic activity of HTSs was postulated to be mainly derived from the unique $3 \mathrm{D}$ hierarchical structures, which raised the activity enhancement in two concrete and proverbial aspects, e.g., specific surface area, porous framework, and the improved light harvesting ability. From the aspects of specific surface area and porous framework, they decreased with increasing the solvothermal reaction time for HTSs (Table S2, Supporting Information), which corresponds to the similar decrease trend of the photodegradation rate. The porous hierarchical structure makes the photocatalyst possess a higher surface-to-volume ratio, assuring a higher total amount of the surface active sites, which enables more reactant molecules to be adsorbed. Also, the hierarchical porous structure not only facilitates the mass transfer rate of pollutant and degradation products but also enables the pollutant molecules to reach into the inner space of the loose hierarchical structures and the pore channels, thus enhancing the photocatalytic efficiency. ${ }^{40,41}$ Regarding the light-harvesting ability, intermeshed nanosheets assembled structures allow for multireflections of trapped incident UVlight among subunits and within their pore channels, thus allowing more efficient use of the irradiated light and increasing quantities of photogenerated electrons and holes that function in photocatalytic reaction, therefore endowing HTSs with greatly enhanced photocatalytic activity. ${ }^{29}$ This was confirmed by the UV-vis adsorption spectra of the HTSs (Figure 6),

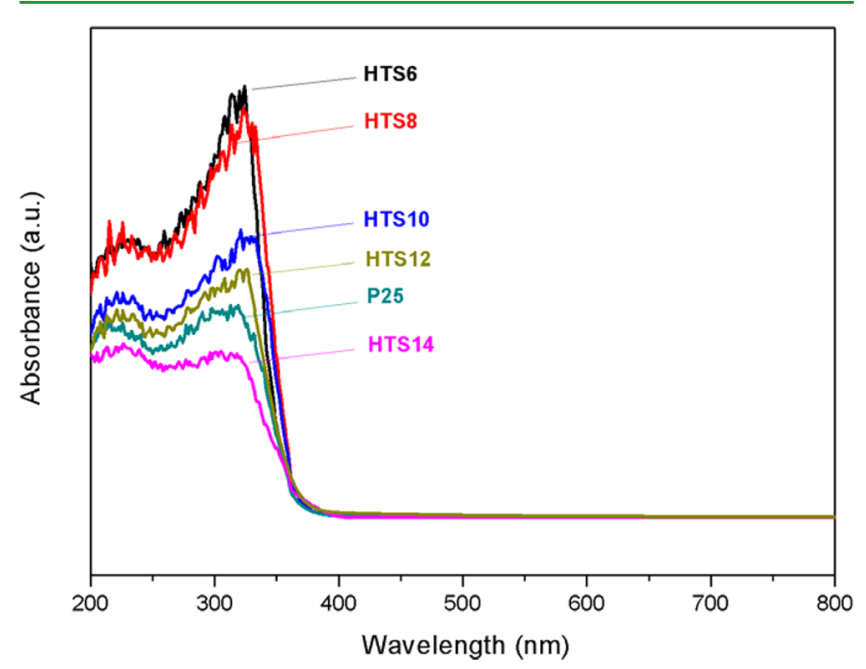

Figure 6. UV-vis spectra of HTSs and Degussa P25.

which clearly demonstrate that the HTSs obtained from longer solvothermal reaction time exhibit weaker absorbance in the UV region. Because it is well-known that the photocatalytic property is largely related to the crystallization degree of anatase, ${ }^{11}$ another reason should be ascribed to the pure anatase phase and better crystallization degree of the samples (Figure S7, Supporting Information). Thus, with the morphology evolving from loose hierarchical flower-like structures to compact particle constructed spheres, the specific surface area, pore volume, and light harvesting ability decreased accordingly. On the basis of the above-mentioned results, it can be concluded that the photocatalytic activity depends strongly on the morphology and structure of the $\mathrm{TiO}_{2}$ catalyst.

\section{CONCLUSIONS}

In summary, a facile one-pot approach has been developed to synthesize 3D hierarchical $\mathrm{TiO}_{2}$ superstructures, by which structure and morphology can be engineered from a poorly crystallized peony-like structure to a semicrystal chrysanthemum-like structure, and eventually to a highly crystallized monocrysal rice-grain built spherical structure. The evolution of the hierarchical structures adopted a four stage mechanism: (1) initial nucleation upon solvothermal reaction and growth of primary particles; (2) random oriented aggregation of nanoparticles forming a rudimentary agglomerate; (3) further growth abided by ordered attachment of crystallites/particles constructing semicrystal nanosheets based hierarchical structures; (4) a moderated proceeding toward equilibrium with the solution adhering to the principle of eliminating high energy 
surfaces and resultant formation of larger well-crystallized particles built spheres. The resulting 3D hierarchical $\mathrm{TiO}_{2}$ superstructures exhibited superior photocatalytic activities over the commercial Degussa P25 nanoparticles, which depend strongly on variation of structures. It is postulated this novel synthesis strategy for $\mathrm{TiO}_{2}$ can be extended to the fabrication of a wide variety of other inorganic materials with evolvable 3D hierarchical structures. More importantly, the proposed formation mechanism also gives an insight into structural evolution during the postformation process.

\section{ASSOCIATED CONTENT}

\section{S Supporting Information}

Details of the Experimental Section, supplementary SEM and TEM images, diameter size distribution, SAED patterns, TG/ DSC curves of the samples, as well as SEM and TEM images, XRD patterns, and XPS spectra of the calcined samples. This material is available free of charge via the Internet at http:// pubs.acs.org.

\section{AUTHOR INFORMATION}

\section{Corresponding Authors}

*G. Li. E-mail: glli@rcees.ac.cn.

*Q. Chen. E-mail: qchen@rcees.ac.cn.

\section{Author Contributions}

The paper was written through contributions of all authors. All authors have given approval to the final version of the paper.

\section{Notes}

The authors declare no competing financial interest.

\section{ACKNOWLEDGMENTS}

This work was jointly supported by the National Natural Science Foundation of China (21207148), the National Basic Research Program of the PRC (2011CB936001), and the National Key Technology R\&D Program (2012BAC07B02).

\section{REFERENCES}

(1) Liu, J.; Qiao, S. Z.; Hartono, S. B.; Lu, G. Q. Monodisperse YolkShell Nanoparticles with a Hierarchical Porous Structure for Delivery Vehicles and Nanoreactors. Angew. Chem., Int. Ed. 2010, 49, 49814985 .

(2) Li, Y.-F.; Liu, Z.-P. Particle Size, Shape and Activity for Photocatalysis on Titania Anatase Nanoparticles in Aqueous Surroundings. J. Am. Chem. Soc. 2011, 133, 15743-15752.

(3) Liu, Z.; Andreev, Y. G.; Armstrong, A. R.; Brutti, S.; Ren, Y.; Bruce, P. G. Nanostructured $\mathrm{TiO}_{2}(\mathrm{~B})$ : The Effect of Size and Shape on Anode Properties for Li-Ion Batteries. Prog. Nat. Sci. 2013, 23, 235-244.

(4) Shang, S. Q.; Jiao, X. L.; Chen, D. R. Template-Free Fabrication of $\mathrm{TiO}_{2}$ Hollow Spheres and Their Photocatalytic Properties. ACS Appl. Mater. Interfaces 2012, 4, 860-865.

(5) Perlich, J.; Memesa, M.; Diethert, A.; Metwalli, E.; Wang, W.; Roth, S. V.; Timmann, A.; Gutmann, J. S.; Mueller-Buschbaum, P. Layer-by-Layer Fabrication of Hierarchical Structures in Sol-Gel Templated Thin Titania Films. Phys. Status Solidi RRL 2009, 3, 118-120.

(6) Chen, X.; Mao, S. S. Titanium Dioxide Nanomaterials: Synthesis, Properties, Modifications, and Applications. Chem. Rev. 2007, 107, 2891-2959.

(7) Wu, D. P.; Zhu, F.; Li, J. M.; Dong, H.; Li, Q.; Jiang, K.; Xu, D. S. Monodisperse $\mathrm{TiO}_{2}$ Hierarchical Hollow Spheres Assembled by Nanospindles for Dye-Sensitized Solar Cells. J. Mater. Chem. 2012, 22, 11665-11671.

(8) Chen, J. S.; Tan, Y. L.; Li, C. M.; Cheah, Y. L.; Luan, D.; Madhavi, S.; Boey, F. Y. C.; Archer, L. A.; Lou, X. W. Constructing
Hierarchical Spheres from Large Ultrathin Anatase $\mathrm{TiO}_{2}$ Nanosheets with Nearly 100\% Exposed (001) Facets for Fast Reversible Lithium Storage. J. Am. Chem. Soc. 2010, 132, 6124-6130.

(9) Yang, W.; Li, J.; Wang, Y.; Zhu, F.; Shi, W.; Wan, F.; Xu, D. A Facile Synthesis of Anatase $\mathrm{TiO}_{2}$ Nanosheets-based Hierarchical Spheres with over $90 \%\{001\}$ Facets for Dye-Sensitized Solar Cells. Chem. Commun. 2011, 47, 1809-1811.

(10) Wu, Y.; Xu, F.; Guo, D.; Gao, Z.; Wu, D.; Jiang, K. Synthesis of $\mathrm{ZnO} / \mathrm{CdSe}$ Hierarchical Heterostructure with Improved Visible Photocatalytic Efficiency. Appl. Surf. Sci. 2013, 274, 39-44.

(11) Zhu, T. J.; Li, J.; Wu, Q. S. Construction of $\mathrm{TiO}_{2}$ Hierarchical Nanostructures from Nanocrystals and Their Photocatalytic Properties. ACS Appl. Mater. Interfaces 2011, 3, 3448-3453.

(12) Brezesinski, T.; Wang, J.; Polleux, J.; Dunn, B.; Tolbert, S. H. Templated Nanocrystal-based Porous $\mathrm{TiO}_{2}$ Films for Next-Generation Electrochemical Capacitors. J. Am. Chem. Soc. 2009, 131, 1802-1809.

(13) Wu, H. B.; Hng, H. H.; Lou, X. W. Direct Synthesis of Anatase $\mathrm{TiO}_{2}$ Nanowires with Enhanced Photocatalytic Activity. Adv. Mater. 2012, 24, 2567-2571.

(14) Grimes, C. A. Synthesis and Application of Highly Ordered Arrays of $\mathrm{TiO}_{2}$ Nanotubes. J. Mater. Chem. 2007, 17, 1451-1457.

(15) O’Regan, B.; Gratzel, M. A Low-Cost, High-Efficiency Solar-Cell Based on Dye-Sensitized Colloidal $\mathrm{TiO}_{2}$ Films. Nature 1991, 353, 737-740.

(16) Hashimoto, K.; Irie, H.; Fujishima, $\mathrm{A}$. $\mathrm{TiO}_{2}$ Photocatalysis: A Historical Overview and Future Prospects. Jpn. J. Appl. Phys. 2005, 44, $8269-8285$.

(17) Fang, B.; Bonakdarpour, A.; Reilly, K.; Xing, Y.; Taghipour, F.; Wilkinson, D. P. Large-Scale Synthesis of $\mathrm{TiO}_{2}$ Microspheres with Hierarchical Nanostructure for Highly Efficient Photodriven Reduction of $\mathrm{CO}_{2}$ to $\mathrm{CH}_{4}$. ACS Appl. Mater. Interfaces 2014, 6, 15488-98.

(18) Galstyan, V.; Comini, E.; Faglia, G.; Sberveglieri, G. $\mathrm{TiO}_{2}$ Nanotubes: Recent Advances in Synthesis and Gas Sensing Properties. Sensors 2013, 13, 14813-14838.

(19) Wu, N.; Wang, J.; Tafen, D. N.; Wang, H.; Zheng, J.-G.; Lewis, J. P.; Liu, X.; Leonard, S. S.; Manivannan, A. Shape-Enhanced Photocatalytic Activity of Single-Crystalline Anatase $\mathrm{TiO}_{2}$ (101) Nanobelts. J. Am. Chem. Soc. 2010, 132, 6679-6685.

(20) Joo, J. B.; Lee, I.; Dahl, M.; Moon, G. D.; Zaera, F.; Yin, Y. D. Controllable Synthesis of Mesoporous $\mathrm{TiO}_{2}$ Hollow Shells: Toward an Efficient Photocatalyst. Adv. Funct. Mater. 2013, 23, 4246-4254.

(21) Liu, S.; Jia, H.; Han, L.; Wang, J.; Gao, P.; Xu, D.; Yang, J.; Che, S. Nanosheet-Constructed Porous $\mathrm{TiO}_{2}-\mathrm{B}$ for Advanced Lithium Ion Batteries. Adv. Mater. 2012, 24, 3201-3204.

(22) Liu, H.; Bi, Z.; Sun, X.-G.; Unocic, R. R.; Paranthaman, M. P.; Dai, S.; Brown, G. M. Mesoporous $\mathrm{TiO}_{2}-\mathrm{B}$ Microspheres with Superior Rate Performance for Lithium Ion Batteries. Adv. Mater. 2011, 23, 3450-3454.

(23) Li, G.; Liu, J.; Jiang, G. Facile Synthesis of Spiny Mesoporous Titania Tubes with Enhanced Photocatalytic Activity. Chem. Commun. 2011, 47, 7443-7445.

(24) Li, H. X.; Bian, Z. F.; Zhu, J.; Zhang, D. Q.; Li, G. S.; Huo, Y. N.; Li, H.; Lu, Y. F. Mesoporous Titania Spheres with Tunable Chamber Structure and Enhanced Photocatalytic Activity. J. Am. Chem. Soc. 2007, 129, 8406-8407.

(25) Mandlmeier, B.; Szeifert, J. M.; Fattakhova-Rohlfing, D.; Amenitsch, H.; Bein, T. Formation of Interpenetrating Hierarchical Titania Structures by Confined Synthesis in Inverse Opal. J. Am. Chem. Soc. 2011, 133, 17274-17282.

(26) Tian, G. H.; Chen, Y. J.; Zhou, W.; Pan, K.; Tian, C. G.; Huang, X. R.; Fu, H. G. 3D Hierarchical Flower-like $\mathrm{TiO}_{2}$ Nanostructure: Morphology Control and Its Photocatalytic Property. CrystEngComm 2011, 13, 2994-3000.

(27) Sun, Z.; Kim, J. H.; Zhao, Y.; Bijarbooneh, F.; Malgras, V.; Lee, Y.; Kang, Y.-M.; Dou, S. X. Rational Design of 3D Dendritic $\mathrm{TiO}_{2}$ Nanostructures with Favorable Architectures. J. Am. Chem. Soc. 2011, 133, 19314-19317.

(28) Ye, M. D.; Zheng, D. J.; Wang, M. Y.; Chen, C.; Liao, W. M.; Lin, C. J.; Lin, Z. Q. Hierarchically Structured Microspheres for High- 
Efficiency Rutile $\mathrm{TiO}_{2}$-based Dye-Sensitized Solar Cells. ACS Appl. Mater. Interfaces 2014, 6, 2900-2908.

(29) Li, G.; Zhang, H.; Lan, J.; Li, J.; Chen, Q.; Liu, J.; Jiang, G. Hierarchical Hollow $\mathrm{TiO}_{2}$ Spheres: Facile Synthesis and Improved Visible-Light Photocatalytic Activity. Dalton Trans. 2013, 42, 85418544.

(30) Ge, S. X.; Wang, B. B.; Li, D. P.; Fa, W. J.; Yang, Z. Y.; Yang, Z.; Jia, G. Y.; Zheng, Z. Surface Controlled Photocatalytic Degradation of $\mathrm{RhB}$ over Flower-like Rutile $\mathrm{TiO}_{2}$ Superstructures. Appl. Surf. Sci. 2014, 295, 123-129.

(31) Nomura, T.; Kousaka, Y.; Alonso, M.; Fukunaga, M.; Satoh, T. A Model for Simultaneous Homogeneous and Heterogeneous Nucleation in the Case of Slow Reaction Rate. J. Colloid Interface Sci. 2000, 221, 195-199.

(32) Cao, S.-W.; Zhu, Y.-J. Surfactant-Free Preparation and Drug Release Property of Magnetic Hollow Core/Shell Hierarchical Nanostructures. J. Phys. Chem. C 2008, 112, 12149-12156.

(33) Zheng, Z.; Huang, B.; Lu, J.; Qin, X.; Zhang, X.; Dai, Y. Hierarchical $\mathrm{TiO}_{2}$ Microspheres: Synergetic Effect of $\{001\}$ and $\{101\}$ Facets for Enhanced Photocatalytic Activity. Chem.- Eur. J. 2011, 17, 15032-15038.

(34) Yu, J. C.; Yu, J. G.; Ho, W. K.; Jiang, Z. T.; Zhang, L. Z. Effects of $\mathrm{F}^{-}$Doping on the Photocatalytic Activity and Microstructures of Nanocrystalline $\mathrm{TiO}_{2}$ Powders. Chem. Mater. 2002, 14, 3808-3816.

(35) Kong, M.; Li, Y.; Chen, X.; Tian, T.; Fang, P.; Zheng, F.; Zhao, $\mathrm{X}$. Tuning the Relative Concentration Ratio of Bulk Defects to Surface Defects in $\mathrm{TiO}_{2}$ Nanocrystals Leads to High Photocatalytic Efficiency. J. Am. Chem. Soc. 2011, 133, 16414-16417.

(36) Yu, J.; Su, Y.; Cheng, B. Template-Free Fabrication and Enhanced Photocatalytic Activity of Hierarchical Macro-/Mesoporous Titania. Adv. Funct. Mater. 2007, 17, 1984-1990.

(37) Yu, J. C.; Zhang, L. Z.; Yu, J. G. Direct Sonochemical Preparation and Characterization of Highly Active Mesoporous $\mathrm{TiO}_{2}$ with a Bicrystalline Framework. Chem. Mater. 2002, 14, 4647-4653.

(38) Kawahara, T.; Konishi, Y.; Tada, H.; Tohge, N.; Nishii, J.; Ito, S. A Patterned $\mathrm{TiO}_{2}$ (Anatase) $/ \mathrm{TiO}_{2}$ (Rutile) Bilayer-Type Photocatalyst: Effect of the Anatase/Rutile Junction on the Photocatalytic Activity. Angew. Chem., Int. Ed. 2002, 41, 2811-2813.

(39) Li, X. X.; Xiong, Y. J.; Li, Z. Q.; Xie, Y. Large-Scale Fabrication of $\mathrm{TiO}_{2}$ Hierarchical Hollow Spheres. Inorg. Chem. 2006, 45, 34933495.

(40) Xiang, Q.; Cheng, B.; Yu, J. Hierarchical Porous CdS Nanosheet-Assembled Flowers with Enhanced Visible-Light Photocatalytic $\mathrm{H}_{2}$-Production Performance. Appl. Catal., B 2013, 138, 299303.

(41) Jiang, S.; Zhou, K.; Shi, Y.; Lo, S.; Xu, H.; Hu, Y.; Gui, Z. In Situ Synthesis of Hierarchical Flower-like $\mathrm{Bi}_{2} \mathrm{~S}_{3} / \mathrm{BiOCl}$ Composite with Enhanced Visible Light Photocatalytic Activity. Appl. Surf. Sci. 2014, 290, 313-319. 